\title{
Development of a Lorentz-Force-Type Slotless Self-Bearing Motor*
}

\author{
Satoshi UENO**, Shin-ichi UEMATSU** and Takahisa KATO** \\ ** Department of Mechanical Engineering, Faculty of Science and Technology, Ritsumeikan University \\ 1-1-1 Nojihigashi, Kusatsu, Shiga 525-8577 Japan \\ E-mail: sueno@se.ritsumei.ac.jp
}

\begin{abstract}
Magnetic bearing motors have advantages such as no friction loss, no abrasion, and lubrication-free operation. However, they are not widely used due to their high cost and large size. In order to solve these problems, a self-bearing motor having a simple structure with distributed windings is proposed. The rotor consists of a permanent magnet and an iron yoke, which rotates in a body. The stator consists of a six-phase distributed winding and is installed between the permanent magnet and the back yoke of the rotor. A Lorentz force, generated by interaction between stator current and permanent magnet field, is used to control the rotation speed and radial position of the rotor. In this study, the rotating torque and bearing force are analyzed theoretically, and methods for their control are discussed. A simple experiment confirms that the proposed self-bearing motor can be realized.
\end{abstract}

Key words : Active Magnetic Bearing, Self-Bearing Motor, Slotless Stator

\section{Introduction}

Recently, the demand for improving the durability and reducing the generated noise of small motors has been increased strongly ${ }^{(1)-(4)}$. A magnetic bearing appears as a proper choice to meet these demands. Various types of small motors using the active magnetic bearing (AMB) or a self-bearing motor have been proposed. However, the AMB is not widely used for its high cost and dimensions are significantly larger than those of mechanical bearings. As a result, the development of a smaller and relatively low cost AMB is desired.

Under such requirements, a six-salient-pole-type AMB has been proposed ${ }^{(2)}$. By reducing the number of salient poles and coils in comparison with the conventional magnetic bearing, AMB tends to have smaller size and lower cost. However, in order to assure of producing large enough bearing force, the structure of this AMB becomes complicated. Therefore, further miniaturization of the six-salient-pole-type AMB is difficult, and a simpler structure is still required.

Currently, a miniaturized brushless DC motor has been developed. Specifically, the miniaturization of a slotless stator-type motor is quite significant, and it is available at the market with the diameter of $2 \mathrm{~mm}$. Since the magnetic bearing has similar structure with the brushless DC motor, the structure of a miniaturized magnetic bearing can be identically designed to that of a micro brushless DC motor. Basing on this feature, we propose a Lorentzforce-type slotless $\mathrm{AMB}^{(4)}$. A schematic drawing of the proposed AMB is shown in Fig.1. A distributed winding without an iron core is installed in the stator, and a cylindrical permanent magnet and yoke are attached to the rotor. Subsequently, a bearing force is generated in accordance with a Lorentz force produced by the stator current and a magnetic field of the permanent magnet. Usually, the force that can be generated by an AMB by employing the Lorentz force is smaller than that generated by a typical magnetic bearing ${ }^{(5)}$. For this reason, it is necessary to supply high current in order to eliminate the unstable force of the permanent magnet. However, this results in an increase in the power consumption. In order to avoid 
this problem, the cylindrical permanent magnet is fixed to the yoke with constant distance. By this way, the unstable force of the permanent magnet becomes zero. Obviously, the proposed magnetic bearing has a simple structure, achieving the goal of miniaturization and cost reduction.

In this study, a self-bearing motor having a structure similar to that of a slotless AMB has been proposed. By combining an AC motor and a magnetic bearing, this magnetic bearing motor can reach very small size and low cost. The bearing force and rotation torque are theoretically analyzed, and methods for their control are derived. The result of the analysis is then confirmed by using a simple experimental setup.

\section{Slotless Self-Bearing Motor}

The structure of the proposed self-bearing motor is shown in Fig. 1. The self-bearing motor carries out position control with two radial degrees of freedom and generates a motor torque. The rotor consists of a cylindrical two-pole permanent magnet and an iron yoke. The airgap between the permanent magnet and iron yoke is invariable to make sure that the unstable attractive force of the permanent magnet becomes zero. The stator consists of a sixphase distributed winding without a core, and being inserted between the permanent magnet and the yoke of the rotor.

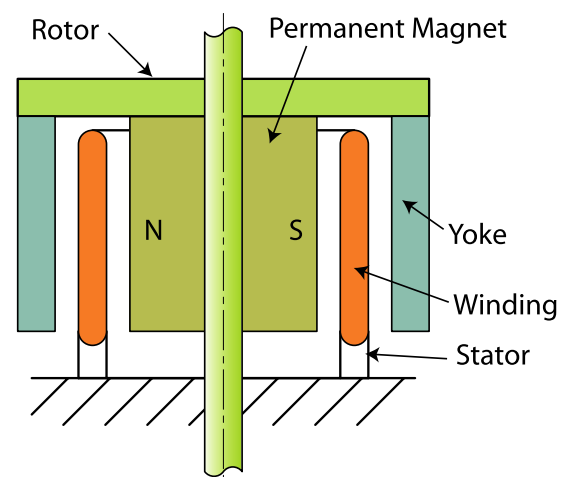

Fig. 1 Schematic of Slotless Self-Bearing Motor

The principle of generating torque and force is shown in Fig. 2. For simplicity, the number of turns of the stator winding is illustrated as one circle, in which indicate the direction of currents. If currents are supplied to the stator winding as illustrated in Fig. 2 (a), a Lorentz force is generated as denoted by the black arrows in the figures. Consequently, a motor torque is generated on the rotor as a reaction force. On the other hand, if currents are supplied as illustrated in Fig. 2 (b), a bearing force is generated as shown in the figure. By inserting both kinds of current as in Fig. 2 (c), the motor torque and bearing force are generated simultaneously. The motor torque and bearing force are controlled by changing the amplitude and the phase of stator currents.

\subsection{Radial Force and Rotating Torque}

Figure 3 describes the coordinate axis used for the analysis of the rotating torque and bearing force. Fig. 3 (a) illustrates the section perpendicular to the motor shaft, while the development along the circumference of the stator winding is shown in Fig. 3 (b). A magnetic flux is positive when travels to the outside of the rotor and vice versa.

The winding has a hexagonal shape as in Fig. 2. Hence, it can be divided in two parts. One is the center of the winding, that is parallel to the axial direction. The other part comprises the top and bottom parts of the winding, and is slanted to turn the direction of the wire. The angular positions of the parallel part are expressed as follows:

$$
\theta_{+ \text {phase }}^{k}=\theta_{0}+\frac{k-1}{3 n} \pi+\frac{2 m}{6} \pi
$$




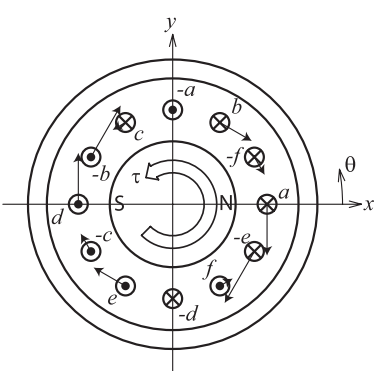

(a) Rotating Torque

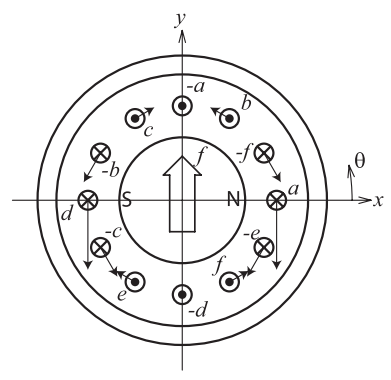

(b) Bearing Force

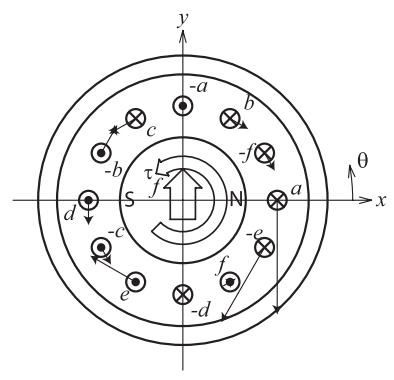

(c) Rotating Torque and Bearing Force

Fig. 2 Generation of Rotating Torque and Bearing Force

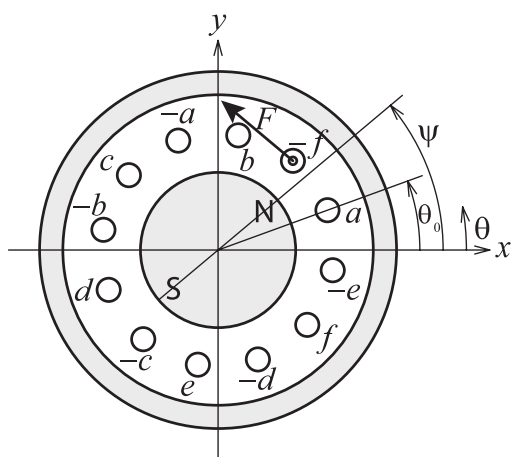

(a) $x-y$

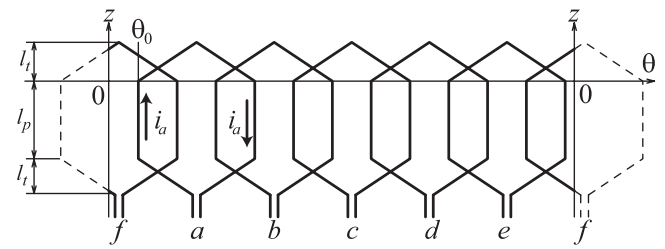

(b) $z-\theta$

Fig. 3 Coordinate Axis

$$
\theta_{- \text {phase }}^{k}=\theta_{0}+\frac{k-1}{3 n} \pi+\frac{2 m+3}{6} \pi
$$

where $m$ is the coefficient corresponding to each phase, $a$-phase $\rightarrow 0$, and $f$-phase $\rightarrow 5, n$ is the total number of turns, $k$ is the turn number, and $\theta_{0}$ is the angular position of the $+a$-phase winding. Where $n$ must be an odd number so that the wires may not overlap.

In order to simplify the analysis, it is assumed that the magnetic field generated by the current is weaker than that generated by the permanent magnet of the rotor, and a sinusoidal waveform distribution of the magnetic flux density is obtained. Then, the magnetic flux density distribution in the airgap is

$$
B_{g}(\theta)=B \cos (\theta-\psi)
$$

where $B$ is the amplitude of the magnetic flux density, and $\psi$ is the angular position of the rotor. The stator currents are the summation of the bearing current and motoring current. Hence, the currents are expressed as follows:

$$
\begin{aligned}
& i_{a, d}=i_{d} \cos (\psi)+i_{q} \sin (\psi) \pm A_{m} \cos \left(\phi_{m}\right) \\
& i_{b, e}=i_{d} \cos (\psi-2 \pi / 3)+i_{q} \sin (\psi-2 \pi / 3) \pm A_{m} \cos \left(\phi_{m}-4 \pi / 3\right) \\
& i_{b, e}=i_{d} \cos (\psi-4 \pi / 3)+i_{q} \sin (\psi-4 \pi / 3) \pm A_{m} \cos \left(\phi_{m}-2 \pi / 3\right)
\end{aligned}
$$

where $i_{d}$ is the direct axis current, $i_{q}$ is the quadric axis current, $A_{m}$ is the amplitude of the motor current, and $\phi_{m}$ is its phase.

First, the case of $n=1$ is considered. The Lorentz-force is calculated by Fleming's law as follows:

$$
f_{p, \pm \text { phase }}=\mp B_{g}\left(\theta_{ \pm \text {phase }}\right) i_{\text {phase }} l_{p}
$$

where $l_{p}$ is the length of the parallel part. The torque produced by the Lorentz force becomes

$$
\tau_{p, \pm \text { phase }}=r f_{p, \pm \text { phase }}
$$


where $r$ is the radius of the winding. The radial force becomes

$$
\begin{aligned}
& f_{p x, \pm \text { phase }}=-f_{p, \pm \text { phase }} \sin \left(\theta_{ \pm \text {phase }}\right) \\
& f_{p y, \pm \text { phase }}=-f_{p, \pm \text { phase }} \cos \left(\theta_{ \pm \text {phase }}\right)
\end{aligned}
$$

The total torque and force are the summation of Eqs. (8), (9) and (10), respectively. Then we have

$$
\begin{aligned}
\tau_{p} & =-3 r l_{p} B A_{m} \sin \left(\phi_{m}-\psi+\theta_{0}+\pi / 4\right) \\
f_{p x} & =3 l_{p} B\left\{i_{d} \sin \left(2 \theta_{0}\right)+i_{q} \cos \left(2 \theta_{0}\right)\right\} \\
f_{p y} & =-3 l_{p} B\left\{i_{d} \cos \left(2 \theta_{0}\right)+i_{q} \sin \left(2 \theta_{0}\right)\right\}
\end{aligned}
$$

The angular position of the turn part can be expressed as follows:

$$
\begin{aligned}
& \theta_{t,+ \text { phase }}(z)=\frac{\pi}{4 l_{t}} z+\frac{2 m}{6}+\theta_{0} \\
& \theta_{t,- \text { phase }}(z)=\frac{2 m+3}{6}-\frac{\pi}{4 l_{t}} z+\theta_{0}
\end{aligned}
$$

where $l_{t}$ is the length of the turn part. The Lorentz force of a small distance in this part is calculated as follows:

$$
\Delta f_{t, \pm \text { phase }}=\mp B_{g}\left(\theta_{t, \pm \text { phase }}(z)\right) i_{\text {phase }} \frac{\Delta z}{\sin \alpha}
$$

where $\alpha$ is a wire angle with its horizontal axis passing through the turn part, and it is expressed as follows:

$$
\alpha=\tan ^{-1} \frac{\theta_{+ \text {phase }}-\theta_{- \text {phase }}}{2 l_{p}} r=\tan ^{-1} \frac{\pi r}{4 l_{p}}
$$

The Lorentz force in the turn part consists of two components force in the axial direction, $\Delta f_{t z}$, and force in the radial direction, $\Delta f_{t t}$. Each force is expressed as follows:

$$
\begin{aligned}
& \Delta f_{t z, \pm \text { phase }}=\Delta f_{t, \pm \text { phase }} \cos \alpha \\
& \Delta f_{t t, \pm \text { phase }}=\Delta f_{t, \pm \text { phase }} \sin \alpha
\end{aligned}
$$

The force in the radial direction becomes

$$
\Delta f_{t t, \pm \text { phase }}=\mp B_{g}\left(\theta_{t, \pm \text { phase }}(z)\right) i_{\text {phase }} \Delta z
$$

and the torque at the turn part is calculated as follows:

$$
\tau_{t, \pm \text { phase }}=\int_{0}^{l_{t}} r f_{t t, \pm \text { phase }}
$$

Then the total torque becomes

$$
\tau_{t}=-\frac{4 r l_{t} B A_{m}}{\pi}(6-3 \sqrt{2}) \sin \left(\phi_{m}-\psi+\theta_{0}+\pi / 4\right)
$$

The radial force of each phase is calculated as follows:

$$
\begin{aligned}
& f_{t x, \pm \text { phase }}=-\int_{0}^{l_{t}} f_{t t \pm \text { phase }} \sin \left(\theta_{t \pm \text { phase }}(z)\right) \\
& f_{t y, \pm \text { phase }}=-\int_{0}^{l_{t}} f_{t t \pm \text { phase }} \cos \left(\theta_{t \pm \text { phase }}(z)\right)
\end{aligned}
$$

The total radial force is the summation of the forces of all phases, then,

$$
\begin{aligned}
& f_{t x}=\frac{6 l_{t} B}{\pi}\left\{i_{d} \sin \left(2 \theta_{0}\right)-i_{q} \cos \left(2 \theta_{0}\right)\right\} \\
& f_{t y}=-\frac{6 l_{t} B}{\pi}\left\{i_{d} \cos \left(2 \theta_{0}\right)+i_{q} \sin \left(2 \theta_{0}\right)\right\}
\end{aligned}
$$


Since the turn part comprises two parts, the total torque and radial force become

$$
\begin{aligned}
& \tau=k_{m} A_{m} \sin \left(\phi_{m}-\psi+\theta_{0}+\pi / 4\right) \\
& f_{x}=-k_{b}\left\{i_{d} \sin \left(2 \theta_{0}\right)-i_{q} \cos \left(2 \theta_{0}\right)\right\} \\
& f_{y}=k_{b}\left\{i_{d} \cos \left(2 \theta_{0}\right)+i_{q} \sin \left(2 \theta_{0}\right)\right\}
\end{aligned}
$$

where

$$
\begin{aligned}
& k_{m}=-\left(3 l_{p}+\frac{8(6-3 \sqrt{2})}{\pi} l_{t}\right) r B \\
& k_{b}=-\left(3 l_{p}+\frac{12}{\pi} l_{t}\right) B
\end{aligned}
$$

For $n=3$, the interval between the phases of the wires is $\pi / 9$. Therefore, the rotating torque can be expressed the summation of Eq. (27), where $\theta_{0}=\theta_{0}-\pi / 9, \theta_{0}$, and $\theta_{0}+\pi / 9$. Then, we have

$$
\tau=\{1+2 \cos (\pi / 9)\} k_{m} A_{m} \sin \left(\phi_{m}-\psi+\theta_{0}+\pi / 4\right)
$$

Similarly, the rotating torque and radial forces in case on $n$ turns are obtained as follows:

$$
\begin{aligned}
& \tau=k_{n m} k_{m} A_{m} \sin \left(\phi_{m}-\psi+\theta_{0}+\pi / 4\right) \\
& f_{x}=-k_{n b} k_{b}\left\{i_{d} \sin \left(2 \theta_{0}\right)-i_{q} \cos \left(2 \theta_{0}\right)\right\} \\
& f_{y}=k_{n b} k_{b}\left\{i_{d} \cos \left(2 \theta_{0}\right)+i_{q} \sin \left(2 \theta_{0}\right)\right\}
\end{aligned}
$$

where

$$
\begin{aligned}
& k_{n m}=1+2 \cos \left(\frac{\pi}{3 n}\right)+2 \cos \left(\frac{2 \pi}{3 n}\right)+\cdots+2 \cos \left(\frac{(n-1) \pi}{6 n}\right) \\
& k_{n b}=1+2 \cos \left(\frac{2 \pi}{3 n}\right)+2 \cos \left(\frac{4 \pi}{3 n}\right)+\cdots+2 \cos \left(\frac{(n-1) \pi}{3 n}\right)
\end{aligned}
$$

\subsection{Closed-Loop Torque Control}

If the angular position of the rotor can be obtained, the stator current can be calculated by Eqs.(4),(5) and (6). Then, assuming $\theta_{0}=0$ and $\phi_{m}-\psi+\theta_{0}+\pi / 4=\pi / 2$, the rotating torque and bearing force become

$$
\begin{aligned}
\tau & =k_{n m} k_{m} A_{m} \\
f_{x} & =k_{n b} k_{b} i_{q} \\
f_{y} & =k_{n b} k_{b} i_{d}
\end{aligned}
$$

Hence, the rotating torque can be controlled by $A_{m}$, and the bearing force can be controlled by $i_{d}$ and $i_{q}$.

The block diagram of a closed-loop torque control is shown in Fig. 4. The radial position controller is a standard PID controller, while the rotating speed controller is a PI controller.

\subsection{Open-loop Torque Control}

If the angular position of the rotor cannot be obtained, the rotating torque can be passively controlled by a current with constant amplitude and frequency. Let $\phi_{m}=\omega t$ and the phase difference between $\phi_{m}$ and $\psi$ denoted by $\Delta \phi_{m}=\phi_{m}-\psi+\pi / 4$ then, we have

$$
\tau=k_{n m} k_{m} A_{m} \sin \left(\Delta \phi_{m}\right)
$$

Hence, the torque is passively controlled by $\Delta \phi_{m}$. If a load torque is small, that is $\Delta \phi_{m} \approx 0, \psi$ can be approximate to $\pi / 4+\phi_{m}$. Therefore the stator current can be calculated by substituting $\psi=\pi / 4+\phi_{m}$ in Eqs. (4), (5) and (6).

The block diagram of an open-loop torque control is shown in Fig. 5. An angular position detector is not required, therefore, the control system becomes simpler. 


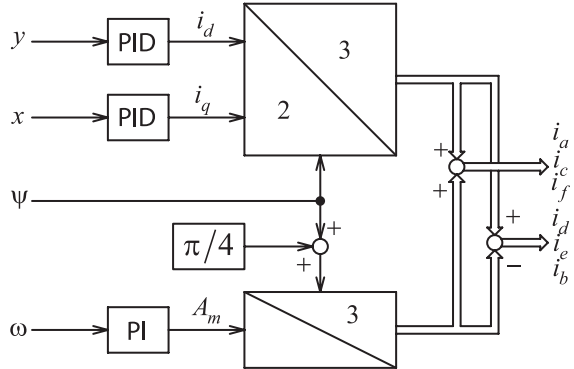

Fig. 4 Closed-loop Torque Control

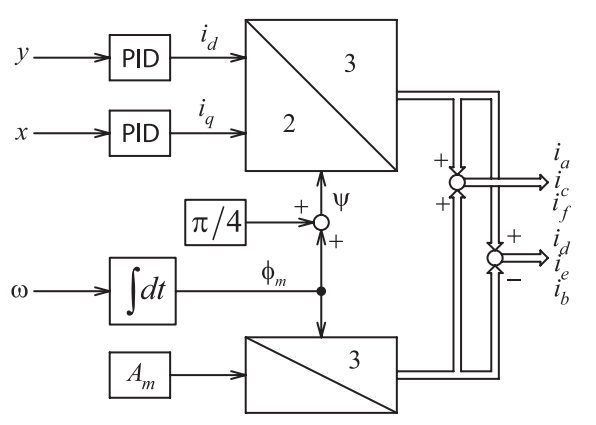

Fig. 5 Open-loop Torque Control

\section{Experimental Results}

\subsection{Experimental Setup}

In order to confirm the above mentioned analysis, a simple experimental setup was designed and tested. The experimental setup is shown in Fig. 6. For simplicity, the rotor top was supported using a ball bearing, therefore, the rotor had three degrees of freedom $x, y$, and around $z$. The self-bearing motor was attached to the lower part of the rotor, and a rotary encoder was attached to the upper part for measuring the angular position of the rotor.

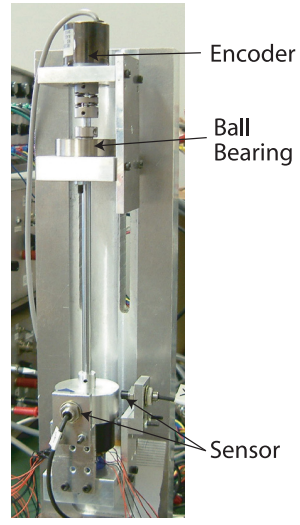

Fig. 6 Experimental Setup
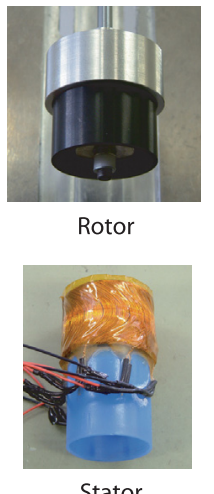

tator

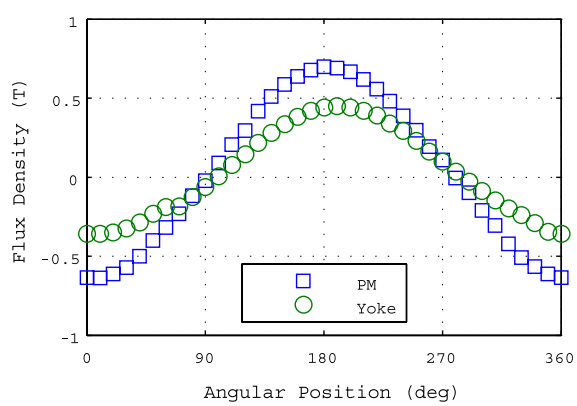

Fig. 7 Flux Density Distribution

The rotor consists of a shaft, a permanent magnet, a back yoke, and one part to fix them together. A two-pole cylindrical neodymium magnet is used. The outer diameter, inner diameter and length of the permanent magnet are $22 \mathrm{~mm}, 8 \mathrm{~mm}$, and $22 \mathrm{~mm}$, respectively. The back yoke is composed of S45C. Its outer and inner diameters are $38 \mathrm{~mm}$ and $34 \mathrm{~mm}$, respectively. The distribution of the magnetic flux density between the permanent magnet and the back yoke is indicated in Fig. 7. The curve drawn by using the squares denotes the result of the flux density measured on the surface of the permanent magnet, and the curve drawn by using the circles denotes the result of the flux density measured on the inner surface of the yoke. From these results, it is confirmed that the distribution of magnetic flux density is sinusoidal, and its maximum amplitude is approximately 0.6T.

The stator consists of the winding and a plastic collar for fixing it. The copper wire has diameter of $0.26 \mathrm{~mm}$ and the number of turns is 55 . The length of the parallel part $l_{p}$ is 6 $\mathrm{mm}$ and the length of the turn part $l_{t}$ is $8 \mathrm{~mm}$. The radius of the stator is $13.5 \mathrm{~mm}$. From Eqs. (30), (31), (36) and (37), the torque coefficient and the bearing force coefficient become $k_{n m} k_{m}=-0.0229(\mathrm{Nm} / \mathrm{A}), k_{n b} k_{b}=-1.33(\mathrm{~N} / \mathrm{A})$, respectively.

The control system is illustrated in Fig. 8. A digital signal processor (DSP) is used for control. The DSP receives signals from two displacement sensors through A/D converters and the angular position of the rotor through an up/down counter. Then the DSP calculates 


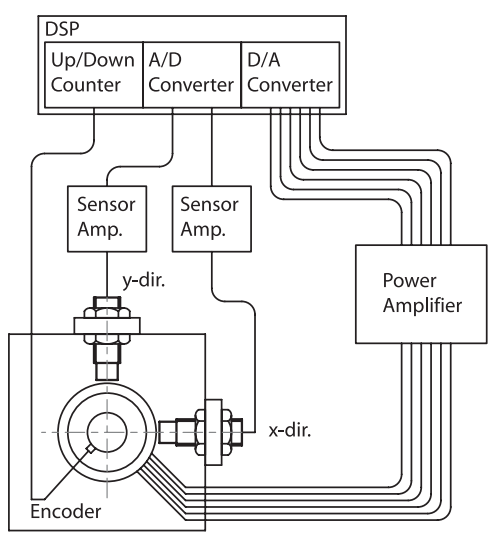

Fig. 8 Control System

the stator reference current and gives its commands to the power amplifier through a D/A converter. This power amplifier is current source amplifier.

\subsection{Motor Torque and Bearing Force}

First, the results of the measured motor torque are shown in Fig. 9. While carrying out the position control, a constant torque current was supplied. The rotation of the rotor was stopped by a force applied by using a force gauge connected to the shaft. Then, the rotating torque was calculated from the value of the force gauge. From this result, it is confirmed that the torque is linear to the torque current.

The measurement results of the bearing force are shown in Fig 10. While carrying out the position control of the rotor, a constant disturbance force was applied in the $x$ direction, and the current was measured when the displacement became zero. The circles and square denote the $i_{d}$ and $i_{q}$ values, respectively. This result indicates that the force is proportional to the current.

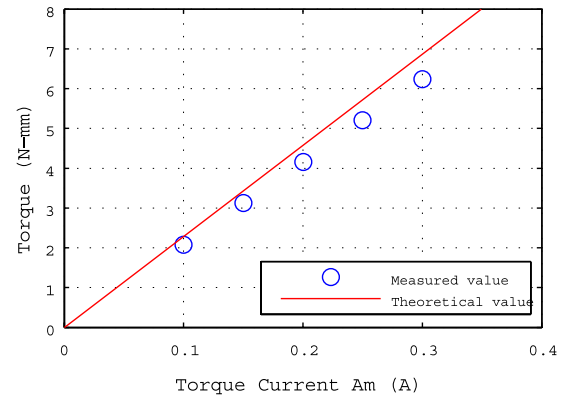

Fig. 9 Rotating Torque

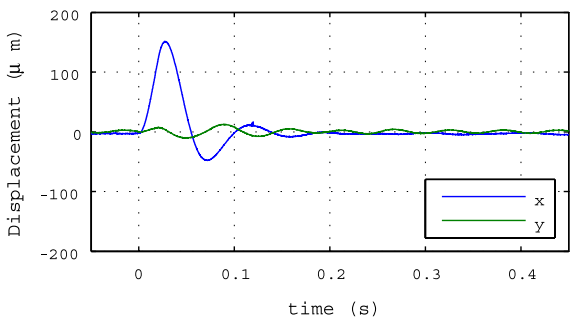

(a) Closed-Loop Torque Control

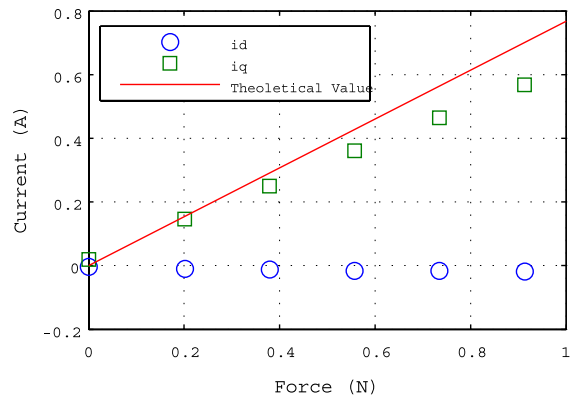

Fig. 10 Bearing Force

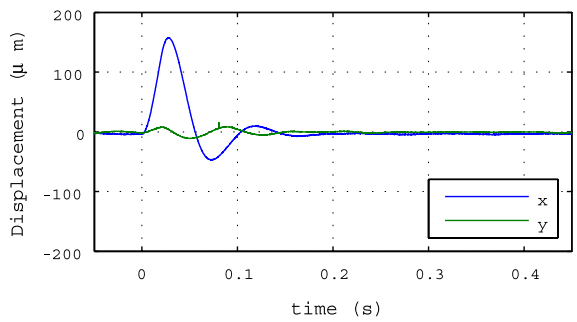

(b) Open-Loop Torque Control

Fig. 11 Impulse Responses 


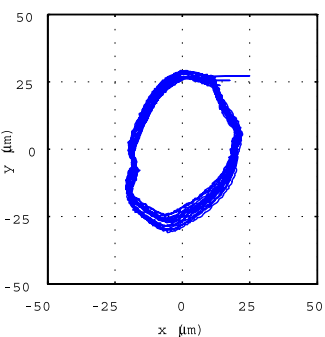

(a) $2,000 \mathrm{rev} / \mathrm{min}$

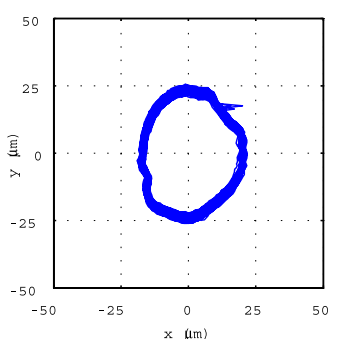

(b) $5,000 \mathrm{rev} / \mathrm{min}$

Fig. 12 Orbit Obtained Using Closed-loop Torque Control

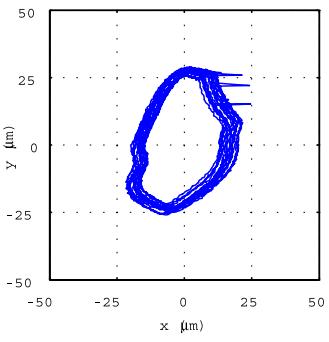

(a) $2,000 \mathrm{rev} / \mathrm{min}$

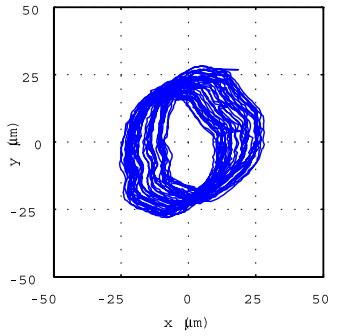

(b) $5,000 \mathrm{rev} / \mathrm{min}$

Fig. 13 Orbit Obtained Using Open-loop Torque Control

\subsection{Impulse Responses}

The impulse responses at $0 \mathrm{rev} / \mathrm{min}$ were measured for confirming the position control. The results are shown in Fig 11. Figures 11 (a) and (b) indicate the results of the impulse responses obtained using the closed-loop torque control and open-loop torque control, respectively. Both these impulse responses settle in $0.2 \mathrm{~s}$, which confirm that position control is stable.

\subsection{Rotation Test}

Figure 12 illustrates a trajectory of the rotor motion in the $x-y$ plane at 2,000 rev/min and $5,000 \mathrm{rev} / \mathrm{min}$, obtained by using the closed-loop torque control. In both cases, the amplitude of vibration is approximately $25 \mu \mathrm{m}$, and the rotor can rotate stably. Figure 13 shows the corresponding results obtained by using the open-loop torque control. In this case, the orbit is unstable in comparison with that obtained by using the closed-loop torque control. In particular, the orbit obtained at $5,000 \mathrm{rev} / \mathrm{min}$ is more unstable than that obtained at 2,000 rev/min. One of the possible reasons for this is that the phase difference $\Delta \phi_{m}$ changes by a perturbation of the rotation torque. This change causes an error of the bearing force, and it influences the radial position control. From these results, it is obvious that the closed-loop torque control is suitable for achieving high-speed rotation. Hence, if the use of an encoder is not possible, a sensorless vector control method should be employed.

Finally, the acceleration test results are shown in Fig. 14. This test was performed using the closed-loop torque control. The rotation speed was changed from 0 to 5,000 rev/min, and the motor current Am, rotation speed, and displacement were measured. The torque current was limited to $0.4 \mathrm{~A}$. The rotation speed becomes $5,000 \mathrm{rev} / \mathrm{min}$ after $2 \mathrm{~s}$. Although the displacement deviates slightly from zero, it is confirmed that the rotor can be supported stably during the acceleration.

\section{Conclusions}

In this study, a novel self-bearing motor is proposed using a slotless distribution winding. The proposed self-bearing motor is capable of being miniaturize because it has a simple structure. The motor torque and bearing force are derived analytically, and methods for the 

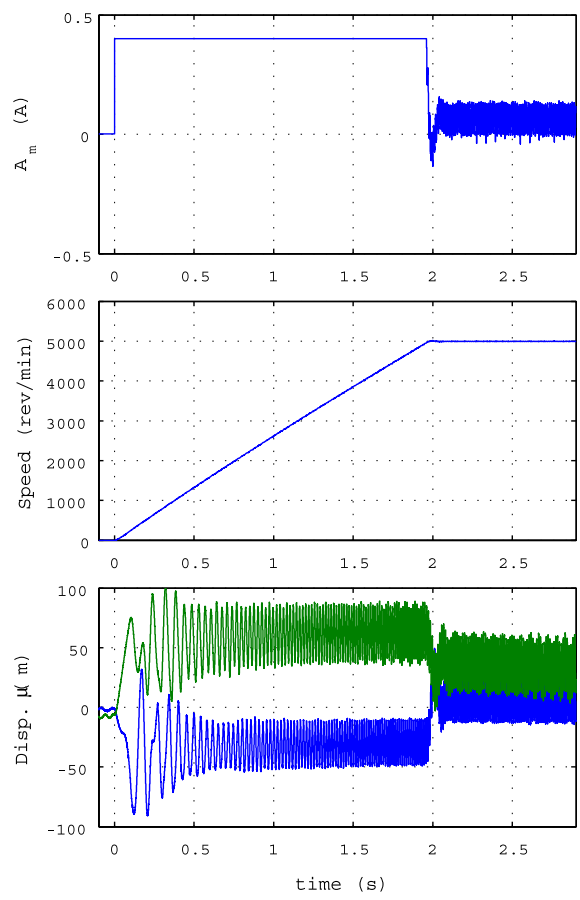

Fig. 14 Acceleration Test

control of the rotation speed and position are discussed. Experimental results show that the proposed self-bearing motor can be realized and it has high potential for high-speed rotation.

\section{References}

( 1 ) Y. Okada et al., JSME Publication on New Technology Series, No. 1, Magnetic Bearings-Fundamental Design and Applications (in Japanese), Yokendo Ltd. Tokyo, (1995).

( 2 ) S. Ueno et al., Development of the Miniature AMB with 6 Concentrated Wound Poles, Proceedings of the 9th International Symposium on Magnetic Bearings, (2004), CDROM

( 3 ) L. Li et al., A Simple and Miniaturized Magnetic Bearing for Cost-Sensitive Applications, Proceedings of 8th International Symposium on Magnetic Bearings, (2002), pp. 561-565.

( 4 ) S. Ueno et al., Development of a Lorentz-force-type Slotless Active Magnetic Bearing, Proceedings of the 10th International Symposium on Magnetic Bearings, (2006), CDROM

( 5 ) T. Tokumoto et al., Comparison between Slot and Slotless Constructions of Lorenz Type Self-bearing Motor, Trans. of JSME, Vol. 68, No. 674 C, pp. 2992-2998, (2002). 